\title{
molecules
}

ISSN 1420-3049

www.mdpi.com/journal/molecules

Article

\section{A Novel Reduplicate Strategy for Tracing Hemostatic Compounds from Heating Products of the Flavonoid Extract in Platycladi cacumen by Spectrum-Effect Relationships and Column Chromatography}

\section{Yeqing Chen ${ }^{1, \dagger}$, Hongli Yu ${ }^{1,2,3, \dagger}$, Hao Wu ${ }^{1,2,3, *}$, Yaozong Pan ${ }^{1}$, Kuilong Wang ${ }^{1}$, Liping Liu ${ }^{1}$, Yangping Jin ${ }^{1}$ and Chengchao Zhang ${ }^{1}$}

1 School of pharmacy, Nanjing University of Chinese Medicine, Nanjing 210023, China; E-Mails: cyqzs88@163.com (Y.C.); yuhongli76@126.com (H.Y.); pan_yaozong@126.com (Y.P.); wjwk1@126.com (K.W.); liuliping1487@163.com (L.L.); yangpingctt@126.com (Y.J.); didiaodexiaojuren@163.com (C.Z.)

2 Jiangsu Key Laboratory of Chinese Medicine Processing, Nanjing University of Chinese Medicine, Nanjing 210023, China

3 Engineering Center of State Ministry of Education for Standardization of Chinese Medicine Processing, Nanjing 210023, China

$\dagger$ These authors contributed equally to this work.

* Author to whom correspondence should be addressed; E-Mail: whao5795@163.com; Tel.: +86-025-8679-8281.

Academic Editor: Derek J. McPhee

Received: 1 August 2015 / Accepted: 11 September 2015 / Published: 17 September 2015

\begin{abstract}
Platycladi cacumen and its processed product have been utilized as a Chinese medicine to treat hemorrhages. In this study, the base peak chromatogram fingerprints of heating products of total flavonoids in Platycladi cacumen were established by high performance liquid chromatography coupled with mass spectroscopy/mass spectroscopy (HPLC-MS/MS), and the hemostatic activities were studied by hemostatic screening tests in vivo. The spectrum-effect relationships between fingerprints and hemostatic activities were analyzed by using canonical correlation analysis to trace the peaks responsible for the significant hemostatic effects. Peak 10 and peak 12 were correlated most closely, thus probably being the main hemostatic compounds. To confirm the reliability of this strategy, the targeted unknown peak was obtained by bioactivity-guided isolation, characterized by
\end{abstract}


MS, ${ }^{1} \mathrm{H}-\mathrm{NMR},{ }^{13} \mathrm{C}-\mathrm{NMR}$, and 2D-NMR spectroscopies, and referred to as cecarbon as a new compound. In addition, the isolated compound exhibited hemostatic effect in a dose-dependent manner with different potencies in vitro and existed in Platycladi cacumen Carbonisatus. A novel dereplication strategy was employed to trace and identify the active compounds of other herbs that have bioactivity enhancement after processing using spectrum-effect relationships and column chromatography.

Keywords: flavonoids; heating products; hemostatic activity; Platycladi cacumen; spectrum-effect relationships

\section{Introduction}

As a traditional Chinese medicine (TCM), Platycladi cacumen (PC) is the dried branches and leaves of Platycladus orientalis (L.) Franco, which belongs to the monotypic genus Platycladus of the Cupressaceae family and has officially been listed in the Chinese Pharmacopoeia [1]. PC has been recorded and summarized in ancient manuscripts, including "Shu Ben Cao Ri Huazi Ben Cao", "Ben Cao Bei Yao" and "De Pei Ben Cao" PC is mainly capable of removing heat from the blood, as well as preventing and treating hemorrhage diseases including hematemesis, epistaxis, hemoptysis, hematochezia, dysfunctional uterine bleeding, and hemorrhoidal bleeding [1,2]. It has been used in contemporary Chinese herbal formulations or dispensed as a medicine, such as Ce Bo San. Phytochemistry research found that flavonoids such as myricetrin, myricetin, quercitrin, quercetin, hinokiflavone, and amentoflavone are the main medicinal components of PC [3-5].

Carbonisatus product is produced by putting original species into a heating container to be processed into a burned black or burned brown surface and a brown or pale brown interior. It has been widely used to treat bleeding diseases. Platycladi cacumen Carbonisatus (PCC), which is the legal processed product of PC [1], has obviously weakened anti-blood-heat activity and enhanced anti-hemorrhagic activity, as suggested by pharmacological and clinical studies [6]. With styptic properties, PCC is commonly used in clinical practice to prevent and to treat various types of hemorrhages including hematochezia, hematemesis, epistaxis, dysfunctional uterine bleeding, and hemorrhoidal bleeding [1]. In addition, PCC has been applied to develop hemostatic agents (e.g., Shi Hui San). Unlike PC, flavonoid aglycones are the main effective components of PCC [7,8]. Despite ongoing endeavors, the components of PCC that show significant hemostatic activity remain unknown.

In this study, we hypothesized that therapeutic effects of PCC can be attributed to certain pharmacological and chemical correlations, particularly transformation of flavonoids during heating. Thus, it is of great significance to combine component analysis with structural identification for the simulated processing (heating) products of total flavonoids in Platycladi cacumen (FPC-N) showing hemostatic effect. However, it is too complicated to predict the activity of each chemical component, and the separation processes are difficult. Therefore, it is important to establish a reasonable spectrum-effect relationship model to analyze and associate the variety of effects caused by the transformations of chemical substances after processing. 
The highly sensitive mass spectrometer (MS), which is often used as the mass analyzer for liquid chromatography (LC), facilitates the detection of low-content compounds that are not detectable using classical methods [9,10]. Recently, high-performance liquid chromatography coupled with mass spectroscopy/mass spectroscopy (HPLC-MS/MS) has been applied to rapidly analyze complex components and chemical transformations [11]. In particular, accurate masses and molecular formulae of untargeted compounds acquired from HPLC-MS/MS, as the most important information, have been used to predict and find the known components [12]. Hemostatic screening tests are classical and common in the laboratory [13], detecting bleeding time (BT), activated partial thromboplastin time (APTT), prothrombin time (PT), thrombin time (TT), fibrinogen content (FIB), euglobulin lysis time (ELT), and platelet aggregation. HPLC-MS/MS [14,15] and hemostatic screening tests [16,17], although widely used alone, have never been combined to analyze the active components in PC and PCC.

The aim of this study was to chemically and biologically characterize hemostatic compounds derived from FPC-N to reveal the material basis for the significantly enhanced hemostatic activity. The chromatograms and spectroscopic data acquired from HPLC-MS/MS were used to establish the fingerprints of FPC-N and to monitor changes on the chemical level during processing. Hemostatic screening tests were selected to evaluate the effects of these samples on blood in vivo. Some quantitative parameters obtained from the hemostatic activities were analyzed using principal component analysis (PCA). Then, the spectrum-effect relationships, combined with HPLC-MS/MS fingerprints and hemostatic activities of different samples, were investigated to trace the peaks responsible for the hemostatic effects, with the help of a canonical correlation analysis (CCA) model. Furthermore, the predicted active peaks in fingerprints were isolated by column chromatography (CC) and their structures were identified by $\mathrm{MS},{ }^{13} \mathrm{C}$ - and ${ }^{1} \mathrm{H}$ - nuclear magnetic resonance (NMR), two-dimensional NMR (2D-NMR), and ultraviolet (UV) spectroscopies. Finally, the hemostatic effect of the isolated compound was verified in vitro.

\section{Results and Discussions}

\subsection{HPLC-MS/MS Analysis}

FPC-N samples were comparatively analyzed using our established HPLC-MS/MS method. The representative base peak chromatograms (BPC) of FPC-N are shown in Figure 1. Fourteen chromatographic peaks were detected and 10 peaks were identified by comparing their retention time and mass spectra with those of reference substances (Table 1). Notably, the relative peak areas of peaks 1, 2, 4-8, and 13-14 decreased with extended heating time. Furthermore, some substances were generated at different heating times, such as peaks 10 and 12, generated observably in FPC-10, and peak 11, generated in FPC-8. The relative heights of peaks 10-12 first increased and then decreased. The relative peak area of each peak that was obtained from XICs is shown in Table 2. Therefore, these seven FPC-N samples, as suggested by the 14 different characteristic peaks, showed significant differences. 


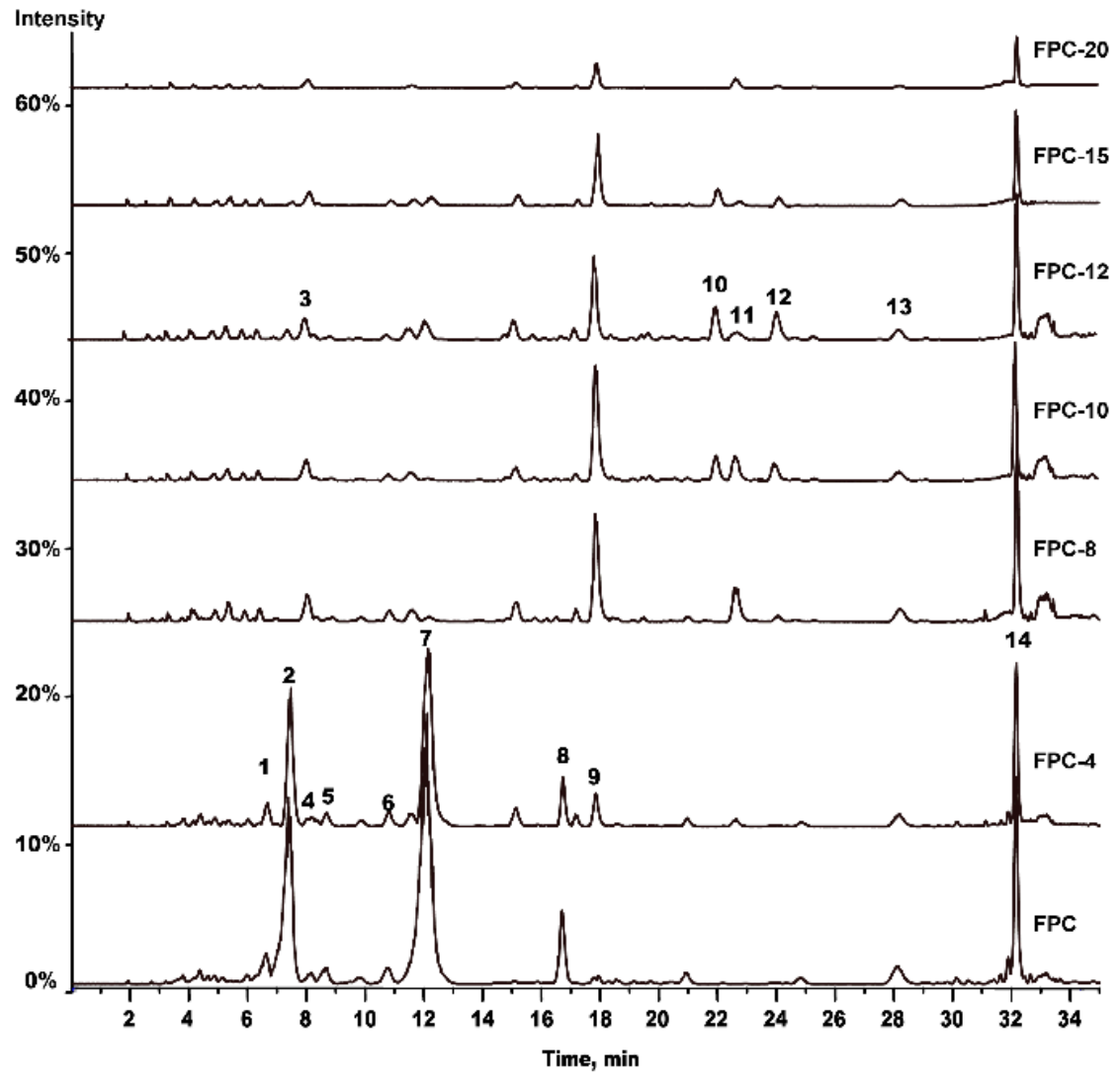

Figure 1. Representative BPC of FPC-N and its six processed products based on HPLC-MS/MS. A total of 14 peaks with large areas and good separation were regarded as "common peaks" in the fingerprints.

Table 1. Characteristics of 14 peaks from FPC-N by HPLC-MS/MS in negative mode.

\begin{tabular}{|c|c|c|c|c|c|}
\hline $\begin{array}{l}\text { Peak } \\
\text { No. }\end{array}$ & $t_{R} / \mathbf{m i n}$ & $\begin{array}{c}{[\mathbf{M}-\mathbf{H}]^{-}} \\
(\text {Error }(\mathbf{p p m}))\end{array}$ & Fragments & Formula & Identification \\
\hline 1 & 6.62 & $509.2050(2.4)$ & $491.1946,367.1411,313.1293,179.0705$ & $\mathrm{C}_{25} \mathrm{H}_{34} \mathrm{O}_{11}$ & Unknown \\
\hline 2 & 7.40 & $463.0864(1.9)$ & $316.0209,287.0176,271.0227,178.9966$ & $\mathrm{C}_{21} \mathrm{H}_{20} \mathrm{O}_{12}$ & Myricetrin \\
\hline 3 & 8.00 & $363.0731(0.3)$ & $331.0469,300.0278,271.0250,178.9979,151.0036$ & $\mathrm{C}_{17} \mathrm{H}_{16} \mathrm{O}_{9}$ & Unknown \\
\hline 4 & 8.17 & $551.2191(1.3)$ & $505.2148,341.1391,329.1398,314.1142$ & $\mathrm{C}_{27} \mathrm{H}_{36} \mathrm{O}_{12}$ & Lyoniside \\
\hline 5 & 8.71 & $463.0894(1.9)$ & $301.0273,271.0240,255.0292,151.0034$ & $\mathrm{C}_{21} \mathrm{H}_{20} \mathrm{O}_{12}$ & Isoquercitrin \\
\hline 6 & 10.85 & $431.0999(1.4)$ & $311.0590,268.0378,240.0417$ & $\mathrm{C}_{21} \mathrm{H}_{20} \mathrm{O}_{10}$ & Apigetrin \\
\hline 7 & 12.10 & $447.0922(1.3)$ & $301.0336,271.0234,255.0283,151.0025$ & $\mathrm{C}_{21} \mathrm{H}_{20} \mathrm{O}_{11}$ & Quercitrin \\
\hline 8 & 16.70 & $431.0984(1.4)$ & $284.0319,255.0290,227.0337$ & $\mathrm{C}_{21} \mathrm{H}_{20} \mathrm{O}_{10}$ & $\begin{array}{l}\text { Kaempferol-3- } \\
\text { O-Rhamnoside }\end{array}$ \\
\hline 9 & 17.82 & $301.0349(0.4)$ & $273.0403,178.9980,151.0037,121.0300,107.0149$ & $\mathrm{C}_{15} \mathrm{H}_{10} \mathrm{O}_{7}$ & Quercetin \\
\hline 10 & 21.90 & $411.0737(1.0)$ & $383.0778,327.0515,261.0410,177.0188,163.0034$ & $\mathrm{C}_{21} \mathrm{H}_{16} \mathrm{O}_{9}$ & Huaicarbon B \\
\hline 11 & 22.60 & $285.0410(-0.8)$ & 239.0348 & $\mathrm{C}_{15} \mathrm{H}_{10} \mathrm{O}_{6}$ & Kaempferol \\
\hline 12 & 24.05 & $327.0718(0.9)$ & $312.0548,281.0813,268.0737,211.0609,165.0683$ & $\mathrm{C}_{19} \mathrm{H}_{20} \mathrm{O}_{5}$ & Unknown \\
\hline 13 & 28.03 & $537.0853(2.6)$ & $417.0628,399.0524,375.0514,331.0614,257.0089,117.0347$ & $\mathrm{C}_{30} \mathrm{H}_{18} \mathrm{O}_{10}$ & Unknown \\
\hline 14 & 32.12 & $537.0836(0.6)$ & $443.0393,417.0602,399.0493,375.0498,331.0594$ & $\mathrm{C}_{30} \mathrm{H}_{18} \mathrm{O}_{10}$ & Amentoflavone \\
\hline
\end{tabular}


Table 2. Relative peak area of each peak of FPC-N.

\begin{tabular}{cccccccccc}
\hline \multirow{2}{*}{ No. } & \multirow{2}{*}{$\mathbf{t}_{\mathbf{R}} / \mathbf{m i n}$} & \multicolumn{8}{c}{ Average Peak Area of Every Peak } \\
\cline { 3 - 10 } & & FPC & FPC-4 & FPC-8 & FPC-10 & FPC-12 & FPC-15 & FPC-20 & C.V. (\%) \\
\hline 1 & 6.62 & 639,370 & 430,101 & 4746 & 0 & 0 & 0 & 0 & 87.68 \\
2 & 7.40 & $3,673,429$ & $2,305,206$ & 16,025 & 0 & 0 & 0 & 0 & 93.86 \\
3 & 8.00 & 12,221 & 147,248 & 202,203 & 177,952 & 173,135 & 85,047 & 47,777 & 91.28 \\
4 & 8.17 & 234,301 & 176,238 & 27,590 & 16,045 & 11,729 & 11,904 & 0 & 101.10 \\
5 & 8.71 & 209,800 & 154,003 & 30,984 & 0 & 0 & 0 & 0 & 92.84 \\
6 & 10.85 & 301,206 & 170,929 & 27,102 & 17,140 & 15,029 & 14,914 & 2146 & 156.31 \\
7 & 12.10 & $4,887,454$ & $2,738,900$ & $3,083,130$ & 0 & 0 & 0 & 0 & 70.39 \\
8 & 16.70 & $1,462,801$ & 843,852 & 15,020 & 80,910 & 33,329 & 14,914 & 0 & 136.94 \\
9 & 17.82 & 30,083 & 580,771 & 762,249 & 944,292 & 606,363 & 426,517 & 135,660 & 59.55 \\
10 & 21.90 & 0 & 0 & 5168 & 212,541 & 260,343 & 102,742 & 1724 & 136.94 \\
11 & 22.60 & 14,020 & 129,650 & 243,812 & 40,920 & 4630 & 2033 & 0 & 155.03 \\
12 & 24.05 & 0 & 0 & 46,391 & 180,799 & 307,302 & 160,890 & 17,284 & 136.94 \\
13 & 28.03 & 250,403 & 264,008 & 220,556 & 189,223 & 142,143 & 112,482 & 96,467 & 59.54 \\
14 & 32.12 & $3,916,454$ & $2,685,583$ & $1,468,588$ & $1,176,757$ & $1,080,712$ & 577,617 & 280,981 & 59.55 \\
\hline
\end{tabular}

\subsection{Hemostatic Activity}

\subsubsection{Quantitative Hemostatic Parameters of FPC-N}

The hemostatic activity was evaluated by measuring BT, APTT, PT, TT, FIB, ELT, and platelet aggregation in vivo. After administration, the hemostatic parameters were measured (Table 3). One-way analysis of variance (ANOVA) based on Student's two-tailed unpaired t-test or Dunnett's test for multiple comparisons test $v s$. control group was used for data analysis. Compared with the blank control group, the FPC-10, FPC-12, and FPC-15 groups significantly reduced BT, PT, and APTT and promoted collagen-induced platelet aggregation. However, TT, FIB, ELT, ADP-induced, and thrombin-induced platelet aggregation were similar in all the experimental groups, suggesting that only the products of FPC, after being heated from 10 to $15 \mathrm{~min}$, had hemostatic effects that facilitated collagen-induced platelet aggregation and plasma coagulation. Even so, the parameters changed following different trends, making it difficult to accurately differentiate the effects of FPC-N on hemostatic activity. Therefore, it was necessary to find out the main parameter(s) that played the most important role in differentiating the FPC-N samples, for which PCA can be used. 
Table 3. Hemostatic parameters of rat plasma affected by FPC-N.

\begin{tabular}{|c|c|c|c|c|c|c|c|c|c|}
\hline \multirow{3}{*}{ Products } & \multicolumn{9}{|c|}{ Parameters } \\
\hline & \multirow[b]{2}{*}{ BT(min) } & \multirow[b]{2}{*}{ PT (s) } & \multirow[b]{2}{*}{ APTT (s) } & \multirow[b]{2}{*}{ TT (s) } & \multirow[b]{2}{*}{ FIB (g/L) } & \multirow[b]{2}{*}{ ELT (min) } & \multicolumn{3}{|c|}{ Maximal Aggregation (\%) } \\
\hline & & & & & & & $\begin{array}{c}\text { ADP } \\
(5 \mu \mathrm{mol} / \mathrm{L}) \\
\end{array}$ & $\begin{array}{c}\text { Collagen } \\
(0.3 \mu \mathrm{g} / \mathrm{mL})\end{array}$ & $\begin{array}{c}\text { Thrombin } \\
\text { (0.1 u) }\end{array}$ \\
\hline Control & $4.96 \pm 0.56$ & $9.87 \pm 0.83$ & $24.77 \pm 0.61$ & $16.88 \pm 0.52$ & $5.21 \pm 0.47$ & $100.08 \pm 8.00$ & $48.80 \pm 3.65$ & $40.35 \pm 3.92$ & $57.90 \pm 4.02$ \\
\hline YNBY & $2.53 \pm 0.35 * *$ & $7.41 \pm 0.53 * *$ & $17.38 \pm 0.64 * *$ & $16.63 \pm 0.39$ & $5.18 \pm 0.32$ & $98.50 \pm 10.10$ & $70.52 \pm 4.15 * *$ & $52.24 \pm 4.08 * *$ & $76.20 \pm 3.82 * *$ \\
\hline FPC & $4.86 \pm 0.35$ & $9.77 \pm 0.46$ & $24.59 \pm 0.56$ & $16.74 \pm 0.46$ & $5.24 \pm 0.36$ & $105.05 \pm 9.35$ & $30.68 \pm 3.82 * *$ & $29.42 \pm 3.05 * *$ & $33.95 \pm 3.66 * *$ \\
\hline FPC-4 & $4.72 \pm 0.34$ & $10.10 \pm 0.37$ & $24.62 \pm 0.51$ & $16.59 \pm 0.50$ & $5.27 \pm 0.34$ & $96.85 \pm 8.00$ & $35.25 \pm 4.28 * *$ & $34.29 \pm 3.92 *$ & $40.50 \pm 3.78 * *$ \\
\hline FPC-8 & $4.50 \pm 0.43$ & $9.53 \pm 0.39$ & $24.03 \pm 0.30$ & $16.92 \pm 0.61$ & $5.28 \pm 0.49$ & $103.60 \pm 7.85$ & $42.35 \pm 3.90$ & $44.15 \pm 4.20$ & $51.35 \pm 3.06$ \\
\hline FPC-10 & $3.63 \pm 0.41 * *$ & $8.88 \pm 0.55 *$ & $21.21 \pm 0.54 * *$ & $16.53 \pm 0.43$ & $5.34 \pm 0.40$ & $99.05 \pm 10.50$ & $48.50 \pm 4.15$ & $49.51 \pm 3.88 *$ & $56.25 \pm 4.08$ \\
\hline FPC-12 & $2.98 \pm 0.65 * *$ & $7.32 \pm 0.63 * *$ & $19.38 \pm 1.00 * *$ & $16.49 \pm 0.43$ & $5.29 \pm 0.43$ & $96.25 \pm 9.90$ & $50.25 \pm 3.85$ & $56.32 \pm 3.75 * *$ & $52.07 \pm 3.65$ \\
\hline FPC-15 & $4.06 \pm 0.45 * *$ & $8.80 \pm 0.61 *$ & $22.83 \pm 0.52 *$ & $16.34 \pm 0.49$ & $5.14 \pm 0.36$ & $101.55 \pm 9.40$ & $48.50 \pm 3.82$ & $46.29 \pm 4.10$ & $57.48 \pm 3.92$ \\
\hline FPC-20 & $4.86 \pm 0.49$ & $9.38 \pm 0.48$ & $23.83 \pm 0.39$ & $16.54 \pm 0.45$ & $5.19 \pm 0.33$ & $97.80 \pm 8.45$ & $47.95 \pm 4.03$ & $43.82 \pm 3.41$ & $55.05 \pm 4.00$ \\
\hline
\end{tabular}

Data represents mean $\pm \mathrm{SD}(n=8)$. The negative control group (Control) was given blank solvent and the positive control group (YNBY) was treated with Yunnan Baiyao.

$* * p<0.01$ vs. control group; * $p<0.05$ vs. control group. 


\subsubsection{Results of PCA}

PCA was used to find out the main parameters that played the most important role in differentiating the FPC-N samples; PCA was used in this part. Nine parameters (BT, PT, APTT, TT, FIB, ELT, ADP-induced, collagen-induced, and thrombin-induced aggregation) of the FPC-N samples were considered as objects and expressed as $X_{1}-X_{9}$. The PCA results showed that the first three principal components $\left(Z_{1}, Z_{2}\right.$ and $\left.Z_{3}\right)$ accounted for $87.06 \%$ of the cumulative contribution rate. The equations of the three principal components from the results of "Component Matrixa" are expressed as follows:

$$
\begin{aligned}
& Z_{1}=-0.888 X_{1}-0.906 X_{2}-0.917 X_{3}-0.587 X_{4}+0.227 X_{5}-0.590 X_{6}+0.756 X_{7}+0.961 X_{8}+0.595 X_{9} \\
& Z_{2}=0.399 X_{1}+0.218 X_{2}+0.357 X_{3}-0.131 X_{4}-0.723 X_{5}+0.010 X_{6}+0.591 X_{7}+0.103 X_{8}+0.719 X_{9} \\
& Z_{3}=0.036 X_{1}+0.114 X_{2}+0.040 X_{3}+0.715 X_{4}-0.513 X_{5}+0.185 X_{6}+0.252 X_{7}+0.204 X_{8}+0.333 X_{9}
\end{aligned}
$$

The absolute values before $\mathrm{X}_{1}, \mathrm{X}_{2}, \mathrm{X}_{3}$, and $\mathrm{X} 8$ in $\mathrm{Z}_{1}, \mathrm{Z}_{2}$, and $\mathrm{Z}_{3}$ are larger, showing that $\mathrm{BT}$, PT, APTT, and collagen-induced aggregation play more important roles in differentiating the FPC samples. Besides, collagen-induced aggregation, which contributes more than BT, PT, and APTT do, was selected to compare the hemostatic effect of FPC-N. As a result, the activities of the tested groups followed a descending order of FPC-12 > FPC-10 > FPC-15 $>$ the other groups that had no significant differences from each other. This might explain why the hemostatic effect of PCC surpassed that of PC and why PCC was required to retain characteristics to some extent.

\subsection{Results of CCA}

CCA was used for the spectrum-effect relationships between the areas of 14 peaks in the TICs and the main hemostatic activity parameters (BT, PT, APTT, and collagen-induced aggregation) (Table 4). The main hemostatic parameters were closely correlated with peaks 10 and 12 in the chemical spectra, i.e., these two peaks might be the main active components of FPC-N affecting hemostatic activity. Peak 10 (huaicarbon B) has been reported to show significant hemostatic activity [18], but further study was needed to identify the structure and confirm the bioactivity of predicted active peak 12 .

Table 4. Correlation coefficients between chromatograms peaks and main hemostatic parameters.

\begin{tabular}{cccccccc}
\hline \multirow{2}{*}{ Parameters } & \multicolumn{7}{c}{ Peak No. (1-7) } \\
\cline { 2 - 8 } & $\mathbf{1}$ & $\mathbf{2}$ & $\mathbf{3}$ & $\mathbf{4}$ & $\mathbf{5}$ & $\mathbf{6}$ & $\mathbf{7}$ \\
\hline BT & 0.5368 & 0.5332 & -0.5557 & 0.5262 & 0.5732 & 0.5116 & 0.6068 \\
PT & 0.5782 & 0.5680 & -0.3176 & 0.5952 & 0.6285 & 0.5478 & 0.6507 \\
APTT & 0.5695 & 0.5630 & -0.4571 & 0.5756 & 0.6202 & 0.5469 & 0.6778 \\
Collagen-Aggregation & -0.8849 & -0.8822 & 0.5644 & -0.8828 & -0.9026 & -0.8702 & -0.8286 \\
\hline \multirow{2}{*}{ Parameters } & $\mathbf{8}$ & $\mathbf{9}$ & $\mathbf{1 0}$ & $\mathbf{1 1}$ & $\mathbf{1 2}$ & $\mathbf{1 3}$ & $\mathbf{1 4}$ \\
\cline { 2 - 8 } & 0.5158 & -0.5721 & -0.9767 & 0.2719 & -0.9861 & 0.3400 & 0.4023 \\
BT & 0.5423 & -0.2718 & -0.8879 & 0.4365 & -0.9588 & 0.5757 & 0.5044 \\
PT & 0.5434 & -0.4706 & -0.9784 & 0.3901 & -0.9729 & 0.4494 & 0.4658 \\
APTT & -0.8703 & 0.5485 & 0.8170 & -0.1752 & 0.8742 & -0.6576 & -0.7950 \\
\hline
\end{tabular}




\subsection{Isolation and Confirmation of the Predicted Active Peaks}

\subsubsection{Isolation and HPLC Analysis}

The eluates containing target peak 12 and bioassay-guided fractions containing peak 12 in FPC-12 extract were further separated by column chromatography (CC), giving the purified compound ( $6.2 \mathrm{mg}$, purity $>92 \%$ ). The purity analysis results are shown in Figure 2.

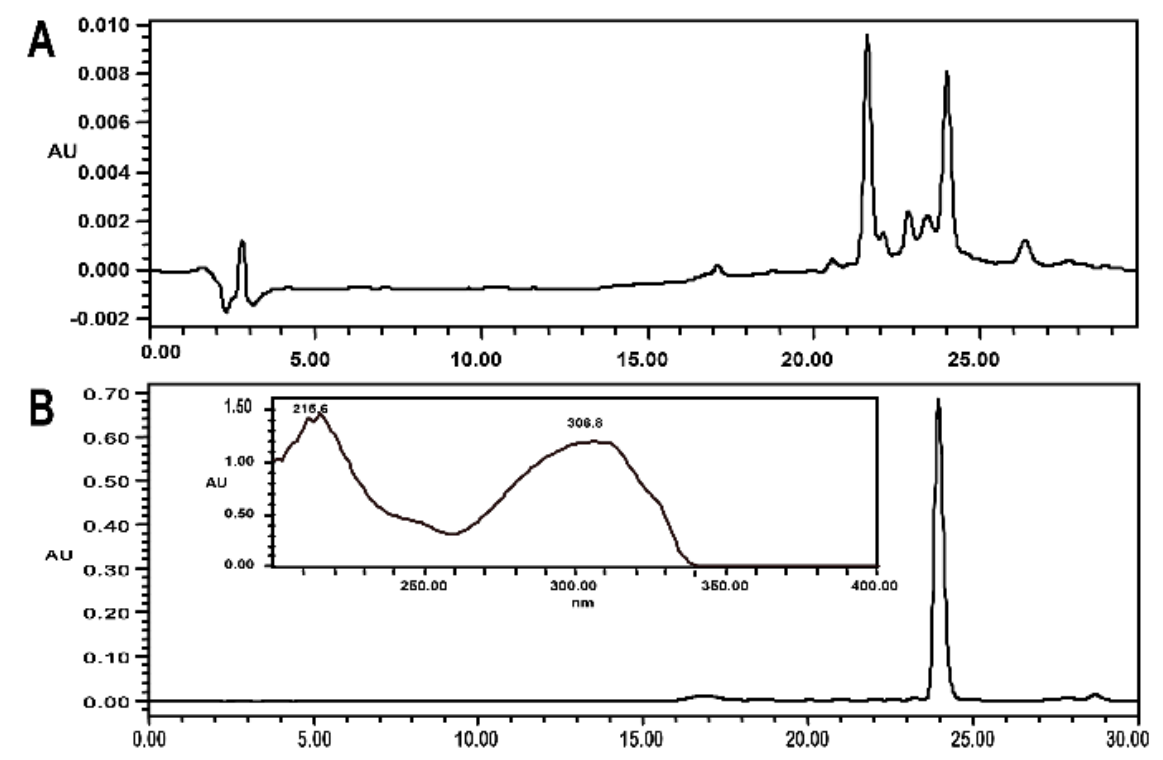

Figure 2. HPLC chromatogram of the fraction containing the peak 12 (A); HPLC chromatogram and UV spectrum of the peak 12 (B).

\subsubsection{Structural Identification}

The chemical structure of the targeted compound (peak 12) was identified according to the MS, 1D-NMR, and 2D-NMR data.

The molecular formula of peak 12 was determined to be $\mathrm{C}_{19} \mathrm{H}_{20} \mathrm{O}_{5}$ on the basis of the molecular ion at $m / z 327.1235[\mathrm{M}-\mathrm{H}]^{-}$by HR-EI-MS. As evidenced by ${ }^{1} \mathrm{H}-\mathrm{NMR},{ }^{13} \mathrm{C}-\mathrm{NMR}$ and HSQC spectra (Table 5), it contained a methoxyl group, a methyl group, three methylene groups, and five methine groups. DEPT, ${ }^{1} \mathrm{H}_{-}{ }^{13} \mathrm{C} \mathrm{HMBC}$, and ${ }^{1} \mathrm{H}-{ }^{1} \mathrm{H}$ COSY were also performed to determine protons linked to individual carbons. HMBC analysis (Figure 3) was employed to confirm the correlation between methylene protons at $\delta_{\mathrm{H}} 2.63, \delta_{\mathrm{H}} 1.75, \delta_{\mathrm{H}} 3.44$ and the quaternary carbon at $\delta_{\mathrm{C}} 137.28$, supporting the linkage of the group to C-6. Methyl protons show HMBC with $\mathrm{C}-1^{\prime}, \mathrm{C}-3, \mathrm{C}-2$, and $\mathrm{C}-4$, indicating that the group was connected to $\mathrm{C}-3$. The COSY spectrum (Figure 3) shows coupling between methylene protons at $\delta_{\mathrm{H}} 1.75$ and those at $\delta_{\mathrm{H}} 2.63$ and $\delta_{\mathrm{H}} 3.44$. A similar shift concerning a similar compound has been reported before by Kimura et al. [19]. By searching databases such as SciFinder Scholar of American Chemical Society, peak 12 was discovered to be a new compound and designated as 2-(4-hydroxy-3-methoxy-phenyl)-5(3-hydroxyl-propyl)-3-methyl-benzofuran-7-ol, referred to as cecarbon. Their structures are shown in Figure 4. 
Table 5. ${ }^{1} \mathrm{H}$ - and ${ }^{13} \mathrm{C}-\mathrm{NMR}$ data of peak 12 .

\begin{tabular}{cccccccc}
\hline Position & $\boldsymbol{\delta}_{\mathbf{H}}$, mult., $\boldsymbol{J}(\mathbf{H z})$ & $\boldsymbol{\delta}_{\mathbf{C}}$ & & Position & $\boldsymbol{\delta}_{\mathbf{H}}, \mathbf{m u l t} ., \boldsymbol{J}(\mathbf{H z})$ & $\boldsymbol{\delta}_{\mathbf{C}}$ & \\
\hline 1 & & & & $2^{\prime}$ & $7.29 \mathrm{~s}$ & 110.32 & $\mathrm{CH}$ \\
2 & - & 150.32 & $\mathrm{C}$ & $3^{\prime}$ & - & 147.74 & $\mathrm{C}$ \\
3 & - & 109.24 & $\mathrm{C}$ & $3^{\prime}-\mathrm{OCH}_{3}$ & 3.84 & 55.68 & $\mathrm{CH}_{3}$ \\
$3-\mathrm{CH}_{3}$ & $2.36 \mathrm{~d}(4.5)$ & 9.29 & $\mathrm{CH}_{3}$ & $4^{\prime}$ & - & 146.86 & $\mathrm{C}$ \\
4 & - & 132.61 & $\mathrm{C}$ & $4^{\prime}-\mathrm{OH}$ & 9.60 & - & - \\
5 & 6.82 & 111.09 & $\mathrm{CH}$ & $5^{\prime}$ & $6.93 \mathrm{~d}(5.1)$ & 115.81 & $\mathrm{CH}$ \\
6 & - & 137.28 & $\mathrm{C}$ & $6^{\prime}$ & $7.22 \mathrm{dd}(6,3.9)$ & 119.59 & $\mathrm{CH}$ \\
7 & 6.58 & 108.89 & $\mathrm{CH}$ & $1^{\prime \prime}$ & $2.63 \mathrm{t}(9.0)$ & 31.81 & $\mathrm{CH}_{2}$ \\
8 & - & 141.63 & $\mathrm{C}$ & $2^{\prime \prime}$ & $1.75 \mathrm{~m}(17.1)$ & 34.81 & $\mathrm{CH}_{2}$ \\
$8-\mathrm{OH}$ & 9.56 & - & - & $3^{\prime \prime}$ & $3.44 \mathrm{t}(7.5)$ & 60.17 & $\mathrm{CH}_{2}$ \\
9 & - & 140.04 & $\mathrm{C}$ & $3^{\prime \prime}-\mathrm{OH}$ & $4.41 \mathrm{~s}$ & - & - \\
$1^{\prime}$ & - & 122.28 & $\mathrm{C}$ & & & & \\
\hline
\end{tabular}

$\mathrm{s}$, singlet; d, doublet; dd, doublet of doublet; $\mathrm{m}$, multiplet; $\mathrm{t}$, triplet.

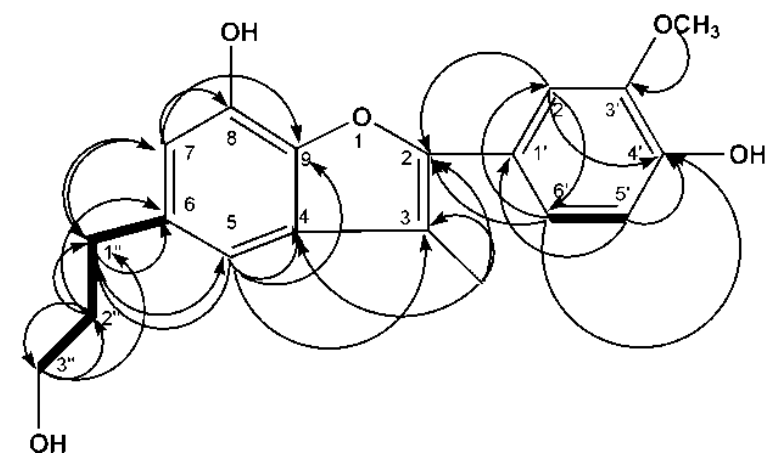

Figure 3. Key HMBC $(\rightarrow)$ and COSY $(-)$ correlations of cecarbon.<smiles>COc1cc(-c2oc3c(O)cc(CCCO)cc3c2C)ccc1O</smiles>

Figure 4. Structure of cecarbon.

\subsubsection{Hemostatic Activity Verification}

The hemostatic activity of peak 12 was investigated in vitro by measuring coagulation parameters (APTT, PT) and the platelet aggregation induced by various agonists. Peak 12 dose dependently decreased APTT (Figure 5) and PT (Figure 6). As shown in Figure 7, the compound promotes collagen-induced platelet aggregation in a dose-dependent manner, but does not significantly affect that induced by ADP or thrombin. These results agreed well with the prediction. Hence, peak 12 in the BPC fingerprints was indeed one of the hemostatic compounds of FPC-N. 


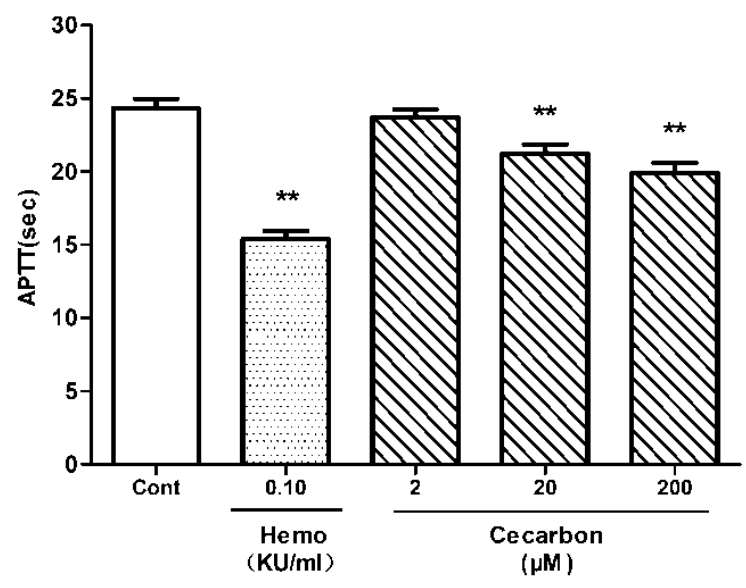

Figure 5. Effect of cecarbon on New Zealand rabbit plasma APTT. Data were expressed as mean $\pm \mathrm{SD}(n=6)$ and $p$-values were calculated using non-parametric repeated measures ANOVA (Friedman test) $(* * p<0.01)$.

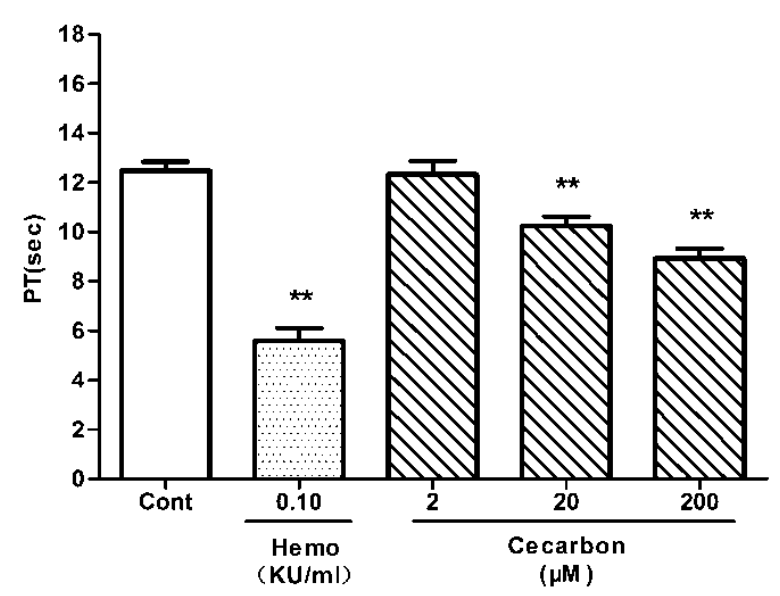

Figure 6. Effect of cecarbon on New Zealand rabbit plasma PT. Data were expressed as mean $\pm \mathrm{SD}(n=6)$. The $p$-values shown were calculated by using non-parametric repeated measures ANOVA (Friedman test) $(* * p<0.01)$.

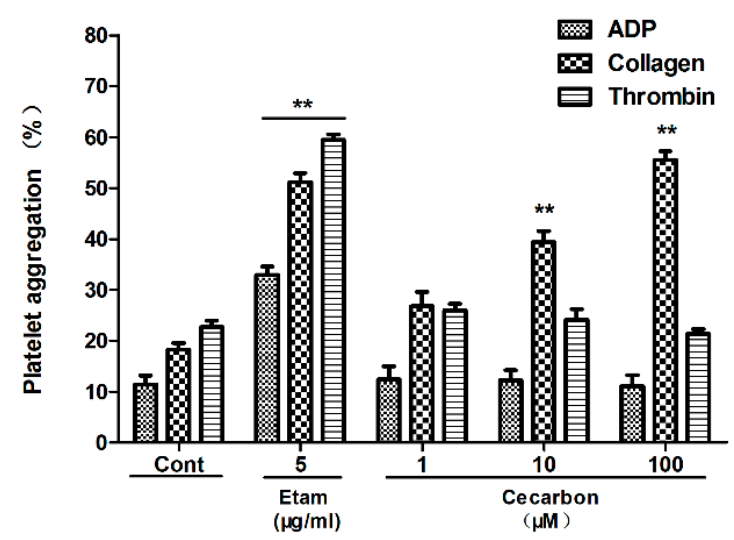

Figure 7. Effect of cecarbon on New Zealand rabbit platelet aggregation induced by ADP, collagen and thrombin. Data were expressed as mean $\pm \mathrm{SD}(n=6)$. The $p$-values were calculated by non-parametric repeated measures ANOVA (Friedman test) $(* * p<0.01)$. 


\subsubsection{Application to the Analysis of PCC Samples}

In TCM practice, PC is processed (heated) in the form of whole branches and leaves rather than a flavonoid extract. To verify whether the resultant new compound obtained from this study is the legitimate active compound formed after PC is processed/heated in practical TCM, the chemical compositions of PCC extract were detected using HPLC. The hemostatic compound was found in PCC (Figure 8). Furthermore, the chemical compositions of FPC-12 were similar to those of PCC extract. Therefore, the subjects of this study were appropriately selected to reflect the reality of TCM practice for PC processing.

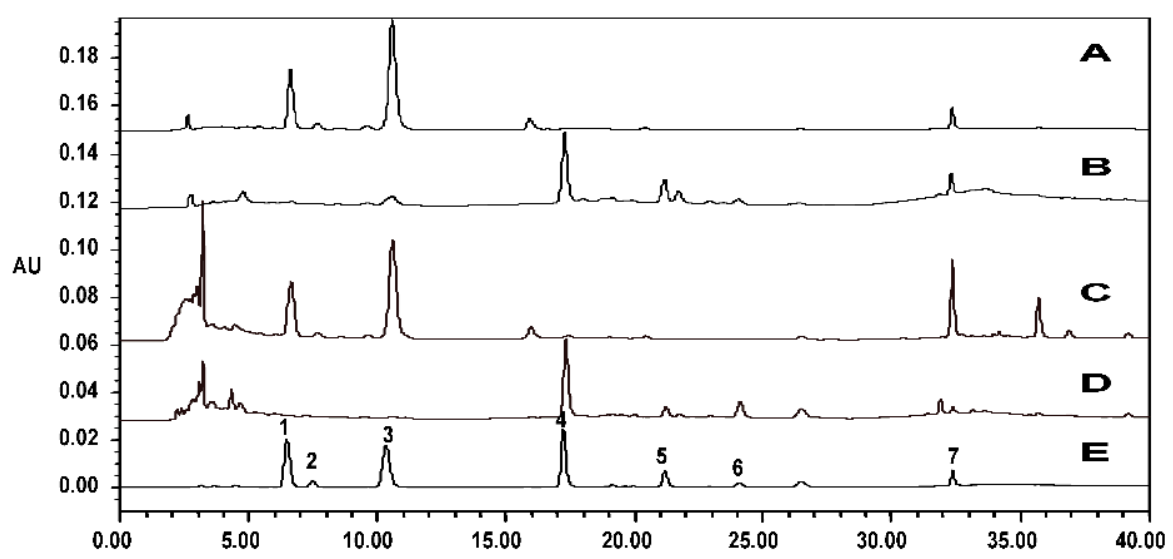

Figure 8. HPLC chromatogram of sample FPC (A); sample FPC-12 (B); sample PC (C); sample PCC (D); and the standard mixtures (E). Seven peaks were identified by comparing with the standard substance: myricetrin (1), isoquercitrin (2), quercitin (3), quercetin (4), huaicarbon B (5), cecarbon (6), and amentoflavone (7).

\section{Experimental Section}

\subsection{Materials and Reagents}

Fresh branches and leaves of Platycladus orientalis (L.) Franco were collected from Heibei Province, China, identified by Professor Chungen Wang (College of Pharmacy, Nanjing University of Chinese Medicine), and then dried. Quercitin, quercetin, myricetrin, afzelin, isoquercitrin, lyoniside, amentoflavone, kaempferol, and apigetrin with 98\% purity were purchased from the National Institutes for Food and Drug Control (Beijing, China). Huaicarbon B with 93\% purity was provided by the Jiangsu Key Laboratory of Chinese Medicine Processing, Nanjing University of Chinese Medicine, China. HPLC-grade methanol and formic acid were purchased from Merck (Darmstadt, Germany). Water for HPLC was purified with a purifier (Research Scientifics Instruments Co. Ltd., Xiamen, China). All solvents used for extraction and CC separation were of analytical grade. Sephadex LH-20 for CC was purchased from GE Healthcare Bio-Science AB. (Uppsala, Sweden), and SiliaSphere PC C18 for CC was from SiliCycle Inc. (Quebec, QC, Canada). Semi-coagulation analyzer (Model LG-PABER-I) was purchased from Steellex Scientific Instrument Company (Beijing, China). A coagulation assay kit was purchased from Nanjing Jiancheng Bioengineering Institute (Nanjing, China). ADP, collagen, and thrombin were purchased from Sigma (St. Louis, MO, USA). 


\section{2. $H P L C-M S / M S$}

\subsubsection{Sample Extraction and Preparation}

FPC was obtained first to prepare FPC-N. PC was extracted with $65 \%$ ethanol $(15: 1, v / w)$ for $2 \mathrm{~h}$, after which the solution was filtered and concentrated to dryness by evaporation to obtain the FPC with $90 \%$ purity (measured by HPLC, the result was provided in Supplementary Materials Figure S1 and Table S1). FPC-N was prepared using single component heating $\left(250{ }^{\circ} \mathrm{C}\right)$ that simulated the carbonization process. FPC $(1.5 \mathrm{~g}, 100 \mathrm{~g}$ crude herb $\times 1.41 \%$ total flavonoid content $) / 90 \%$ purity) powders were put into a 100-mL crucible and heated in a muffle furnace for $4 \mathrm{~min}$ (FPC-4), $8 \mathrm{~min}$ (FPC-8), $10 \mathrm{~min}$ (FPC-10), $12 \mathrm{~min}$ (FPC-12), $15 \mathrm{~min}$ (FPC-15), and $20 \mathrm{~min}$ (FPC-20), respectively, to obtain FPC-N.

The FPC-N samples were ground to fine powders in a mill. Accurately weighed powders (10 mg) were dissolved with $10 \mathrm{~mL}$ of methanol, and sonicated for $30 \mathrm{~min}$. The supernatant was filtered through a $0.22-\mu \mathrm{m}$ syringe filter before being analyzed. Fingerprints were established based on the peak areas and retention times of the main components in FPC-N.

\subsubsection{Instruments and HPLC-MS/MS Conditions}

HPLC-MS/MS analysis was performed on 20DXR HPLC system (SHIMADZU, Japan). Separation was performed with a Dubhe C18 column $(250 \mathrm{~mm} \times 4.6 \mathrm{~mm}, 5.0 \mathrm{~mm}$; Hanbon, China). The mobile phase was a mixture of methanol (A) and $0.1 \%$ formic acid-water (B), with an optimized linear gradient elution as follows: 0-10 min: 48\% A; 10-15 min: 48\%-60\% A; 15-25 min: 60\% A; 25-30 min: 60\%-80\% A; 30-40 min: $80 \%$ A. The flow rate was $1.0 \mathrm{~mL} / \mathrm{min}$. The injection volume was $10 \mu \mathrm{L}$. The flow rate was set at $1.0 \mathrm{~mL} / \mathrm{min}$ with split ratio of $1: 1$. The column temperature was set at $30^{\circ} \mathrm{C}$.

Mass spectrometry was performed on the Triple TOFTM 5600 equipped with an electrospray ionization (ESI) source (AB SCIEX, Redwood City, CA, USA). Samples were analyzed in negative ion modes to provide complementary information for structural identification using the following conditions: mass range, $m / z$ 100-1500; nebulizer gas (gas 1) of 55 psi; heater gas (gas 2) of 55 psi; curtain gas of 35; ion spray voltage of $7 \mathrm{eV}$; turbo spray temperature (TEM) of $550{ }^{\circ} \mathrm{C}$; declustering potential (DP) of $60 \mathrm{~V}$ for MS; declustering potential (DP) of $100 \mathrm{~V}$ for MS/MS; collision energy (CE) of 10 for MS; collision energy (CE) of 35 and collision energy spread (CES) of 15 for MS/MS; nitrogen was kept as the nebulizer and auxiliary gas. The data were acquired and analyzed using PeakView ${ }^{\mathrm{TM}}$ software, and the accurate mass and composition for the precursor and fragment ions were analyzed. Chromatographic peaks in the samples were identified by accurate mass information and retention times of targeted reference substances. Peak areas values were obtained using the XIC Manager in PeakView ${ }^{\mathrm{TM}}$ software. Extracted ion chromatograms (XICs) were automatically generated for each targeted analyte and compared against a user-defined threshold.

\subsection{Hemostatic Assays}

\subsubsection{Sample Preparation and Animals}

SPF Wistar rats of both sexes weighing 220-250 g were purchased from the experimental animal center of Nanjing University of Chinese Medicine (Nanjing, China). All animal experiments were 
approved by the Animal Ethics Committee of Nanjing University of Chinese Medicine, and were carried out according to the Guidelines for the Care and Use of Laboratory Animals. Rats were housed in a conventional animal facility with free access to standard laboratory chow and tap water and were allowed to acclimatize for at least one week before experiment. Rats fasted overnight with free access to water before experimentation. The FPC-N samples were ground into fine powders and were uniformly dispersed in the $0.5 \%$ sodium carboxymethyl cellulose (CMC-Na) for animal tests.

\subsubsection{Experimental Procedure}

The animals were divided randomly into nine groups with eight animals in each group. The negative control group was given blank solvent and the positive control group was treated with Yunnan Baiyao (YNBY, $300 \mathrm{mg} / \mathrm{kg}$ ). The test groups 3-9 were treated with FPC, FPC-4, FPC-8, FPC-10, FPC-12, FPC-15, and FPC-20 $(300 \mathrm{mg} / \mathrm{kg})$ respectively through oral gavage once a day. After the fifth administration, blood was obtained from common carotid aortas. Hemostatic tests (BT, APTT, PT, TT, FIB, ELT, and platelet aggregation) were conducted according to kit instructions and the methods described in previous literatures [20-22].

\subsection{Data Analysis}

\subsubsection{Principal Component Analysis (PCA)}

As a standard data reduction technique, PCA extracts data, removes redundant information, and visualizes the main relationships that exist among a large number of variables in terms of a smaller number of potential factors without losing much information [23]. In this study, PCA was performed on the quantitative hemostatic parameters of FPC-N that were obtained from hemostatic assays using SPSS 16.0 statistics software (SPSS for Windows 16.0, SPSS Inc., Chicago, IL, USA).

\subsubsection{Canonical Correlation Analysis (CCA)}

The basic idea of CCA is to study the correlation between two sets of variations [24]. CCA can reveal and catch the most information between them [25]. CCA was herein used for the spectrum-effect relationships between the peak areas in HPLC-MS/MS XICs and the quantitative hemostatic parameters using SPSS 16.0 statistical software (SPSS for Windows 16.0, SPSS Inc.).

\subsection{Isolation and Confirmation of the Predicted Active Peaks}

\subsubsection{Extraction and Isolation}

FPC-12 powders $(25 \mathrm{~g})$ were mixed with $1 \mathrm{~L}$ of methanol and sonicated for $1 \mathrm{~h}$ at $70{ }^{\circ} \mathrm{C}$. This step was then repeated twice for complete extraction. The total extracts were combined, filtered, and evaporated with a rotary evaporator. The concentrated solution $(25 \mathrm{~mL})$ was subjected to $\mathrm{C} 18 \mathrm{CC}$ and eluted stepwise individually with $1 \mathrm{~L}$ of $20 \%, 40 \%, 60 \%, 80 \%$, or $100 \%$ methanol. Then the eluent was collected at $400 \mathrm{~mL}$ intervals and analyzed by HPLC. The eluents with the same compositions were combined according to the HPLC analysis results. The pooled fractions were tested for coagulation and platelet aggregation assays. Finally, the predicted and active peaks (eluted with $80 \%$ methanol) were 
further applied to CC on a Sephadex LH-20 column (1200 $\mathrm{mm} \times 10 \mathrm{~mm}$ i.d.) with dichloromethane-methanol $(1: 1, v / v)$. To isolate each compound from the corresponding fractions, HPLC analysis was performed on a Waters e2695 Separations Module system equipped with a Photodiode Array Detector. Dubhe C18 column $(250 \mathrm{~mm} \times 4.6 \mathrm{~mm}, 5.0 \mathrm{~mm})$, an analytical column of reverse-phase HPLC, was eluted gradiently with a mobile phase of methanol (A)/0.1\% formic acid (B) (0-10 min: 48\% A; 10-15 min: 48\%-60\% A; $15-25 \mathrm{~min}: 60 \% \mathrm{~A} ; 25-30 \mathrm{~min}: 60 \%-80 \% \mathrm{~A} ; 30-40 \mathrm{~min}: 80 \% \mathrm{~A}$ ) at a flow rate of $1 \mathrm{~mL} / \mathrm{min}$ while maintaining the column temperature at $30{ }^{\circ} \mathrm{C}$.

\subsubsection{Structural Identification}

NMR ( ${ }^{1} \mathrm{H}-\mathrm{NMR},{ }^{13} \mathrm{C}-\mathrm{NMR}$, HSQC, HMBC, COSY, and DEPT) spectra for the compound were recorded using NMR spectrometer systems (Bruker Avance DRX-500, Zurich, Switzerland) with DMSO- $d_{6}$ as solvents, operated at $500 \mathrm{MHz}$. The chemical shifts were reported in ppm, and coupling constants $(J)$ were in $\mathrm{Hz}$.

\subsubsection{Hemostatic Activity Verification}

Hemostatic activity of the compound was verified by pro-coagulation and plasma platelet aggregation assays in vitro. The compound was dissolved in DMSO to prepare final concentrations of $0,1,10$, or $100 \mu \mathrm{M}$ for platelet aggregation assay and $0,2,20$, or $200 \mu \mathrm{M}$ for plasma pro-coagulation assay. Etamsylate (Etam) and haemocoagulase (Haem) were selected as positive controls, and 2\% DMSO was used as the blank control (Cont). ADP, collagen, and thrombin (final concentrations: $0.05 \mu \mathrm{mol} / \mathrm{L}$, $0.03 \mu \mathrm{g} / \mathrm{mL}$, and $0.01 \mathrm{u} / \mathrm{mL}$, respectively) were selected as agonists for the platelet aggregation test. All measurements were determined according to kit instructions and the methods described in previous literature [26].

\subsubsection{Application to the Analysis of PCC Samples}

The chemical compositions of PCC extract were identified using HPLC, as described in Section 3.5.1. The PCC extract solution was prepared as described in the Pharmacopoeia of the People's Republic of China [1]. In brief, accurately weighed powders $(0.5 \mathrm{~g})$ were suspended in $50 \mathrm{~mL}$ of methanol, and sonicated for $30 \mathrm{~min}$.

\section{Conclusions}

Spectrum-effect relationship was established first by combining HPLC-MS/MS fingerprints of FPC-N with hemostatic tests to reveal the potential bioactive compounds responsible for the tested hemostatic activity. A new hemostatic compound, which was generated after the flavonoid extract of PC was heated, was discovered and named cecarbon, which may be valuable for gaining insight into the unique herb processing practice in TCM. This study provides an available reference model for tracing the active compounds of other herbs that have stronger bioactivities after processing.

\section{Supplementary Materials}

Supplementary materials can be accessed at: http://www.mdpi.com/1420-3049/20/09/16970/s1. 


\title{
Acknowledgments
}

This study was financially supported by Major Projects of Science and Technology during the 12th Five-year Plan Period (2012ZX09304005-003) and Research and Innovation Projects for College Graduates of Jiangsu Province (CXLX13-598). The authors would like to acknowledge the Jiangsu Key Laboratory of Chinese Medicine Processing, Nanjing University of Chinese Medicine for providing the laboratory equipment used in this study.

\section{Author Contributions}

Hao $\mathrm{Wu}$ and Hongli Yu designed and directed the research; Yeqing Chen, Yaozong Pan, Kuilong Wang, Liping Liu, Yangping Jin, and Chenchao Zhang performed the research and analyzed the data; Yeqing Chen and Hongli Yu wrote the paper. All authors read and approved the final manuscript.

\section{Conflicts of Interests}

The authors declare no conflict of interests.

\begin{abstract}
Abbreviations
Platycladi Cacumen (PC); Platycladi Cacumen Carbonisatus (PCC); base peak chromatograms (BPC); high-performance liquid chromatography-mass spectroscopy/mass spectroscopy (HPLC-MS/MS); heating products of total flavonoids in Platycladi Cacumen (FPC-N); nuclear magnetic resonance (NMR); mass spectroscopy (MS); Traditional Chinese Medicine (TCM); activated partial thromboplastin time (APTT); prothrombin time (PT); thrombin time (TT); fibrinogen content (FIB); rat-tail bleeding time (BT); euglobulin lysis time (ELT); the flavonoid extract from Platycladi Cacumen (FPC); FPC was heated for $4 \mathrm{~min}$ (FPC-4), 8 min (FPC-8), $10 \mathrm{~min}$ (FPC-10), $12 \mathrm{~min}$ (FPC-12), $15 \mathrm{~min}$ (FPC-15), or $20 \mathrm{~min}$ (FPC-20); principal component analysis (PCA); canonical correlation analysis (CCA); column chromatography (CC); ultraviolet spectroscopy (UV); extracted ion chromatograms (XICs); Yunnan Baiyao (YNBY); Etamsylate (Etam); Haemocoagulase (Haem); blank control (Cont).
\end{abstract}

\section{References}

1. Commission, C.P. The Pharmacopoeia of the People's Republic of China; Chemical Industry Press: Beijing, China, 2010; Volume 1, pp. 200-201.

2. Kim, T.H.; Li, H.; Wu, Q.; Lee, H.J.; Ryu, J.H. A new labdane diterpenoid with anti-inflammatory activity from Thuja orientalis. J. Ethnopharmacol. 2013, 146, 760-767.

3. Lu, Y.H.; Liu, Z.Y.; Wang, Z.T.; Wei, D.Z. Quality evaluation of Platycladus orientalis (L.) Franco through simultaneous determination of four bioactive flavonoids by high-performance liquid chromatography. J. Pharm Biomed Anal. 2006, 41, 1186-1190.

4. Pelter, A.; Warren, R.; Hameed, N.; Khan, N.U.; Ilyas, M.; Rahman, W. Biflavonyl pigments from Thuja orientalis (Cupressaceae). Phytochemistry 1970, 9, 1897-1898.

5. Khabir, M.; Khatoon, F.; Ansari, W.H. Phenolic constituents of Platycladus orientalis. Curr. Sci. 1985, 54, 1180-1185. 
6. Liu, C.; Liu, J.; Zhang, L.; Li, S.F.; Zhang, L.N.; Ding, A.W.; Yu, B. Comparison on hemostasis of Platycladi cacumen before and after processing on blood heat and hemorrhage syndrome rat model. Chin. Tradit. Herb. Drugs 2014, 45, 668-672.

7. Wu, H.E.; Zhen, H.H.; Wei, Z.Y.; Chen, C.L. Determination of quercetin and kaempferol in Platycladus orientalis carbonisatus by RP-HPLC. Lishizhen Med. Mater. Med. Res. 2009, 20, 354-356.

8. Sun, L.L.; Yang, S.B.; Jiang, B.; Zhong, F.X.; Shi, D.H. The effect of processing on Platycladi cacumen chemical components. Chin. Tradit. Patent Med. 2006, 28, 821-823.

9. Figueiredo-González, M.; Cancho-Grande, B.; Boso, S.; Santiago, J.L.; Martínez, M.C.; Simal-Gándara, J. Evolution of flavonoids in Mourton berries taken from both bunch halves. Food Chem. 2013, 138, 1868-1877.

10. Li, Z.F.; Wang, Y.W.; Ouyang, H.; Lu, Y.; Qiu, Y.; Feng, Y.L.; Jiang, H.L.; Zhou, X.; Yang, S.L. A novel dereplication strategy for the identification of two new trace compounds in the extract of Gastrodia elata using UHPLC/Q-TOF-MS/MS. J. Chromatogr. B 2015, 988, 45-52.

11. Peng, Y.; Zhao, L.; Lin, D.J.; Liu, Y.; Zhang, M.; Song, S.J. The chemical constituents' determination of the different processed products of Anemarrhena asphodeloides Rhizomes by high-performance liquid chromatography quadrupole time-of-flight mass spectrometry (HPLC-Q-TOF-MS/MS). Biomed. Chromatogr. 2015, doi:10.1002/bmc.3575.

12. Yang, H.; Lee, D.Y.; Kang, K.B.; Kim, J.Y.; Kim, S.O.; Yoo, Y.H.; Sung, S.H. Identification of ginsenoside markers from dry purified extract of Panax ginseng by a dereplication approach and UPLC-QTOF/MS analysis. J. Pharm. Biomed. Anal. 2015, 109, 91-104.

13. Lippi, G.; Favaloro, E.J. Laboratory hemostasis: milestones in Clinical Chemistry and Laboratory Medicine. Clin. Chem. Lab. Med. 2013, 51, 91-97.

14. Song, Z.L.; Hashi, Y.K.; Sun, H.Y.; Liang, Y.; Lan, Y.X.; Wang, H.; Chen, S.Z. Simultaneous determination of 19 flavonoids in commercial trollflowers by using high-performance liquid chromatography and classification of samples by hierarchical clustering analysis. Fitoterapia 2013, 91, 272-279.

15. Shaw, L.H.; Chen, W.M.; Tsai, T.H. Identification of Multiple Ingredients for a Traditional Chinese Medicine Preparation (Bu-yang-huan-wu-tang) by Liquid Chromatography Coupled with Tandem Mass Spectrometry. Molecules 2013, 18, 11281-11298.

16. Tutanc, M.; Arica, V.; Motor, S.; Basaralan, F.; Erden, E.S.; Ozturk, O.H.; Zararsiz, I.; Aydin, M. Effects of erdosteine on hemostasis: An experimental study. Hum. Exp. Toxicol. 2012, 31, 574-578.

17. Song, Q.L.; Wang, S.S.; Zhao, W.J. Total steroidal alkaloids from Veratrum patulum L. Inhibit platelet aggregation, thrombi formation and decrease bleeding time in rats. J. Ethnopharmacol. 2012, 141, 183-186.

18. Chen, Y.Q.; Yu, H.L.; Wu, H.; Pan, Y.Z.; Wang, K.L.; Liu, L.P.; Jin, Y.P.; Zhang, C.C. Tracing novel hemostatic compounds from heating products of total flavonoids in Flos sophorae by spectrum-effect relationships and column chromatography. J. Sep. Sci. 2015, 38, 1691-1699.

19. Kimura, H.; Tokuyama, S.; Ishihara, T.; Ogawa, S.; Yokota, K. Identification of new flavonol $O$-glycosides from indigo (Polygonum tinctorium Lour) leaves and their inhibitory activity against 3-hydroxy-3-methylglutaryl-CoA reductase. J. Pharm. Biomed. Anal. 2015, 108, 102-112. 
20. Dejana, E.; Callioni, A.; Quintana, A.; Gaetano, G. Bleeding time in laboratory animals. II-A comparison of different assay conditions in rats. Thromb. Res. 1979, 15, 191-197.

21. Roshal, M. Laboratory Techniques in Fibrinolysis Testing. In Transfusion Medicine and Hemostasis-Clinical and Laboratory Aspects; Shaz, B.H., Hillyer, C.D., Roshal M., Abrams, C.S., Eds.; Elsevier: Amsterdam, The Netherlands, 2013; pp. 881-886.

22. Mustard, J.F.; Perry, D.W.; Ardlie, N.G.; Packham, M.A. Preparation of suspensions of washed platelets from humans. Br. J. Hameatol. 1972, 22, 193-204.

23. Zheng, Q.F.; Zhao, Y.L.; Wang, J.B.; Liu, T.T.; Zhang, B.; Gong, M.; Li, J.Y.; Liu, H.H.; Han, B.; Zhang, Y.M.; et al. Spectrum-effect relationships between UPLC fingerprints and bioactivities of crude secondary roots of Aconitum carmichaelii Debeaux (Fuzi) and its three processed products on mitochondrial growth coupled with canonical correlation analysis. J. Ethnopharmacol. 2014, 153, 615-623.

24. Gumus, E.; Kursun, O.; Sertbas, A.; Ustek, D. Application of canonical correlation analysis for identifying viral integration preferences. Bioinformatics 2012, 28, 651-655.

25. Liu, X.; Wang, X.L.; Wu, L.; Li, H.; Qin, K.M.; Cao, H.; Pei, K.; Liu, T.; Cai, B.C. Investigation on the spectrum-effect relationships of Da-Huang-Fu-Zi-Tang in rats by UHPLC-ESI-Q-TOF-MS method. J. Ethnopharmacol. 2014, 154, 606-612.

26. Liu, L.; Duan, J.A.; Tang, Y.; Guo, J.M.; Yang, N.Y.; Ma, H.Y.; Shi, X.Q. Taoren-Honghua herb pair and its main components promoting blood circulation through influencing on hemorheology, plasma coagulation and platelet aggregation. J. Ethnopharmacol. 2012, 139, 381-387.

Sample Availability: Samples of the compounds are not available from the authors.

(C) 2015 by the authors; licensee MDPI, Basel, Switzerland. This article is an open access article distributed under the terms and conditions of the Creative Commons Attribution license (http://creativecommons.org/licenses/by/4.0/). 